\title{
Low voltage electroosmotic pump for high density integration into microfabricated fluidic systems
}

\author{
Friedjof C. A. Heuck • Urs Staufer
}

Received: 13 August 2010/Accepted: 22 November 2010/Published online: 13 January 2011

(C) The Author(s) 2011. This article is published with open access at Springerlink.com

\begin{abstract}
A low voltage electroosmotic (eo) pump suitable for high density integration into microfabricated fluidic systems has been developed. The high density integration of the eo pump required a small footprint as well as a specific on-chip design to ventilate the electrolyzed gases emerging at the platinum $(\mathrm{Pt})$ electrodes. For this purpose, a novel liquid-gas (lg) separator was invented. This lg-separator separated the gas bubbles from the liquid and guided them away from the eo pump. Its operational principle was solely based on the geometry of tapered sidewalls. An eo pump sandwiched by two $\mathrm{lg}$ separators (microchannels in the range of $10 \mu \mathrm{m}$, footprint of $100 \mu \mathrm{m} \times 15 \mu \mathrm{m}$ ) was experimentally investigated. The lg-separator was able to reliably separate and ventilate an emerging gas flow of $2 \mathrm{pl} \mathrm{s}^{-1}$. The eo pump achieved flow rates of $50 \mathrm{pl} \mathrm{s}^{-1}$ at actuation voltages of $5 \mathrm{~V}$.
\end{abstract}

Keywords Electroosmosis $\cdot$ Microfluidic $\cdot$ Gas-liquid interface $\cdot$ Micropump

\section{Introduction}

Electroosmotic (eo) pumps have attracted considerable attention within the recent years (Ghosal 2004; Laser and Santiago 2004; Morf et al. 2001; Guenat et al. 2001; Seibel et al. 2008; Brask et al. 2005; Hug et al. 2005; Takamura

F. C. A. Heuck - U. Staufer

Micro and Nanoengineering Laboratory, $3 \mathrm{mE}$, Delft University of Technology, Mekelweg 2, 2628 CD Delft, The Netherlands

F. C. A. Heuck $(\varangle) \cdot$ U. Staufer

DIMES, Delft University of Technology,

Feldmannweg 17, 2628 CT Delft, The Netherlands

e-mail: f.c.a.heuck@tudelft.nl et al. 2003), due to their ability to provide pulsation free flow without any moving parts. These active electrically controlled pumps are highly suitable for the integration into complex fluidic systems (Seibel et al. 2008). A key challenge of the design of eo pumps is the coupling of the electrical current into the ionic solution, which is commonly done with electrodes made out of Pt. However, in that case, the electron transfer is linked to electrolyzing the solvent and gases are formed. These gases emerge as bubbles and may block the conductive liquid-path between the electrodes and, hence, inhibit a further actuation of the pump. Furthermore, once a gas bubble is fully developed and stretches out over the complete microchannel crosssection, they become almost immobile, since in these dimensions, capillary forces from the liquid-gas interface are the dominate force. It is, therefore, imperative to keep these bubbles away from the eo pump and the functional liquid part of the fluidic system.

Several methods to avoid mixing the electrolyzed gases with the functional fluidic system have been investigated and reported in literature. Brask et al. (2005) used a hybrid approach for the current coupling by assembling an ion exchange membrane between the electrodes and the eo active microchannel. These membranes were permeable for the ionic current and reliably stopped the electrolyzed gases to enter the fluidic system. In order to omit bulky fluidic connections or a membrane, Hug et al. (2005) used a design of different electrical resistances within the fluidic system. In their work, the electrodes were placed into top open reservoirs which were connected to wide microchannels. These wide microchannels connected the submicron wide eo pump. A voltage drop between the electrodes led to a proportionally large voltage drop over the eo pump, due to its comparatively large electrical resistance. Another approach of omitting bulky fluidic 
connections and thus allowing further miniaturizing of the eo pump was the integration of photocurable gel inside the fluidic system, as shown by Takamura et al. (2003). This photocurable gel served as a salt bridge towards $\mathrm{Ag} / \mathrm{AgCl}$ electrodes, which avoided gas bubbles formation. This process allowed a miniaturization but it required the more complex integration of a photocurable gel.

All the above mentioned eo pump designs avoided the interaction of liquid and gas inside the microchannels. For cases, where gas and liquid do get into direct contact inside microchannels, strategies to handle and separate segmented liquid-gas flows have been developed (Guenther et al. 2005), which might be adapted to the situation of the eo pump. A theoretical description of gas bubbles inside microchannel constrictions has been presented by Jensen et al. (2004). In their work, it is mentioned that such constrictions exert considerable Young-Laplace pressure on gas bubbles. Paust et al. (2009) used a version formed by tapered microchannel sidewalls to passively guide gases for direct methanol fuel cells. With the current solutions, a high density integration of eo pumps into microfluidic systems is not possible. A design suitable for the same purpose in the case of an eo micropump, its implementation and analysis is presented in this paper. The mixing of electrolyzed gases within the functional fluidic system is avoided by a novel concept of a liquid-gas (lg) separator. This lg-separator is placed between the electrodes and the eo pump and it drives the emerging gas bubbles away via an exhaust microchannel. The strong capillary forces from the liquid-gas interface in the micrometer ranged microchannels enable the lg-separator to reliably work solely by its geometry.

This paper is structured as follows: a model of the eo pump embedded in a fluidic system is developed. In detail, the physical background of the lg-separator is explained. Then, the model of the lg-separator is assembled together with the eo pump and the connecting microchannels to a fluidic system. In order to extract the relevant design parameters for the fabrication, the pressures and flows within the fluidic system are determined. Finally, the eo fabrication process for the eo pump is outlined and the experimental validation of the design is presented.

\section{Electroosmotic pump with liquid-gas-separator}

\subsection{Model of the liquid-gas-separator}

\subsubsection{Geometry of the liquid-gas-separator and general assumptions}

The lg-separator consisted of a tapered sidewall and an electrode integrated into the bottom of the microchannel, as shown in Fig. 1. It is integrated into the fluidic system with its narrow side, the inlet, oriented towards the eo pump and with its larger side, the outlet, facing the exhaust. The electrode of the eo pump, at which the gas bubble forms, is located inside the lg-separator. This bubble is geometrically confined by the bottom and the sidewalls. It will be shown in the following section that in a tapered microchannel the bubble moves towards the wider side and, hence, away from the electrode. The bubble growth is analytically described in three dimensions. Behind the electrode pointing towards the outlet of the lg-separator, the bubble may stretch over the complete cross-section of the microchannel and may touch the top. At this moment, the water column within the microchannel is separated and any pressure change between the inlet and the outlet of the lg-separator acts directly on the bubble, respectively, on their two separate menisci. The lg-separator compensates for these external pressure changes by its backpressure. In order to model the backpressure, it is assumed that the microchannel is much higher than wide and, hence, the curvature of the meniscus is completely defined by the width (Han et al. 2006), which reduces the model to two dimensions.

The formation and movement of the gas bubbles was modeled in a quasi static approximation. In addition, the processes were assumed to be isothermal, since any heat would be quickly removed by thermal conduction through the surrounding bulk silicon ( $\mathrm{Si}$ ). The following parameters were used in the model: the viscosity of the liquid (water) $\eta=1 \mathrm{mPas}$ and the surface tension of the water-air interface $\gamma_{l a}=73 \mathrm{~mJ} \mathrm{~m}^{-2}$. The contact angle for the
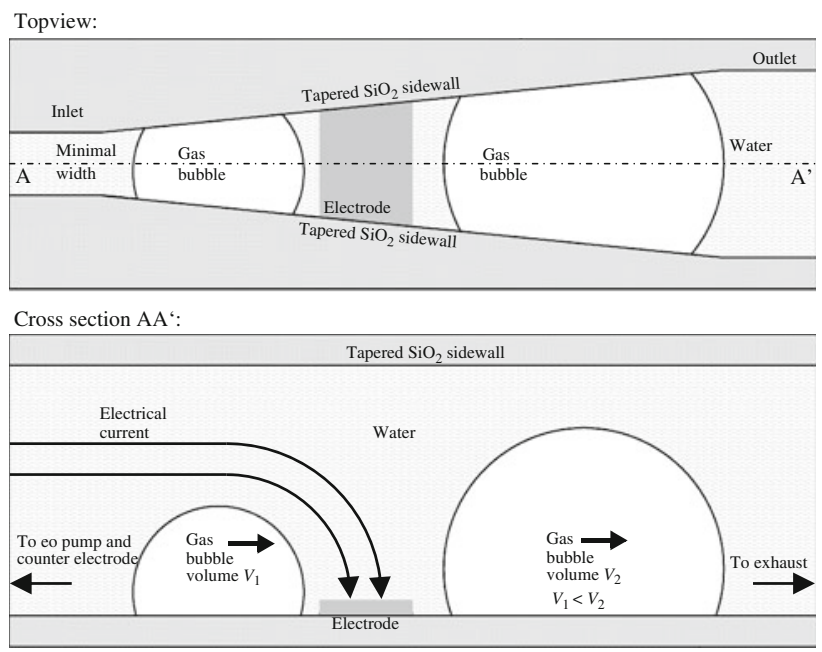

Fig. 1 Sketch of the $1 g$-separator. It enables the coupling of electrical current into the solution and the separation of the emerging electrolyzed gas bubbles from the functional liquid fluidic system. Its operation is based on tapered sidewalls which drive the electrolyzed gas bubbles with increasing bubble volume away from the electrode towards the exhaust 
silicon dioxide $\left(\mathrm{SiO}_{2}\right)$ microchannel and water was measured under static conditions to be $\theta_{s}=45^{\circ}$. The contact angle hysteresis for the observed flow rates over the thermally grown $\mathrm{SiO}_{2}$ surfaces was measured to be $5^{\circ}$, i.e., a receding contact angle of $\theta_{r}=42^{\circ}$ and advancing contact angle of $\theta_{a}=47^{\circ}$.

\subsubsection{Bubble development at the electrode}

Electrolysis initiates the bubble growth at the electrode. Assuming that the electrolyzed gas conserves its internal energy $U_{i n t}$, according to the ideal gas law, enables us to define its internal pressure $p_{i n t}$ and its volume $V$

$U_{\text {int }}=p_{\text {int }} V$.

In the absence of any external pressures, the internal pressure $p_{\text {int }}$ needs to match the Young-Laplace pressure $p_{c}, p_{\text {int }}=p_{c}$, which describes the effect of the liquid gas surface tension $\gamma_{l a}$ confining the liquid-gas interface of the bubble. Due to the properties of a liquid, the shape of the liquid-gas interface is spherical with the radius $R$. The capillary Young-Laplace pressure $p_{c}$ can then be described by

$p_{c}=\frac{\gamma_{l a}}{R}$.

At the bottom, the bubble encounters the transition between three different interfacial energies, the solid-liquid, the liquid-air, and the solid-air interfacial energy. At this intersection, Young's equation demands that the angle enclosed by the tangent of the liquid-gas interface and the bottom equals the static contact angle $\theta_{s}$. Figure 2a shows the spherical bubble at the bottom of the microchannel. The $z$ coordinate defines the distance from the bottom of the microchannel. The height of the bubble center is $R_{h}=R$ $\cos \theta_{s}$. Figure $2 \mathrm{~b}$ shows the horizontal cross-section of the spherical bubble in its center. Due to symmetry, the bubble's horizontal center is also in the horizontal center of the microchannel. The bubble center's distance $R_{w}$ from the tapered sidewall is defined as $R_{w}=w \cos \beta$, where $w$ is half of the microchannel width and $\beta$ the taper angle of the microchannel. The taper of the microchannel is defined as $w=w_{0}+x \tan \beta$, where $w_{0}$ and $x$ denote, half of the initial microchannel width at the electrode and the distance of the bubble center from the electrode, respectively. The direction $x$ is defined to origin at the smaller edge of the electrode and to point towards the outlet of the $\mathrm{lg}$-separator.

This initial bubble $B_{0}$ can grow until it touches the tapered microchannel sidewall. Its radius $R_{0}$ then equals the distance between the center of the bubble and the sidewall $R_{0}=w_{0} \cos \beta$ at the smaller side of the electrode $x_{0}=0$. The internal energy $U_{0}$ of the initial bubble $B_{0}$ can be calculated to

$U_{0}=p_{0} V_{0}=\frac{\gamma_{l a}}{R_{0}} \int_{-R_{0}}^{R_{0} \cos \theta_{s}} \int_{0}^{2 \pi} \int_{0}^{\sqrt{R_{0}^{2}-z^{2}}} r \mathrm{~d} r \mathrm{~d} \omega \mathrm{d} z$.

As soon as, the initial bubble $B_{0}$ touches the sidewall, two more transition points between three different interfacial energies occur, at which the Young's boundary condition requires the contact angle $\theta_{s}$. Hence, the bubble needs to change its shape to a new first bubble $B_{1}$ and as a consequence its position $x_{0} \rightarrow x_{1}$ shifts within the microchannel. Figure 3a shows the geometry of the first bubble. The shape of the first bubble $B_{1}$ remains spherical, due to the absence of any external pressures. The radius $R_{1}$ of the first bubble can be calculated to

$R_{1}=\frac{R_{w}}{\cos \theta_{s}}=\left(w_{0}+x \tan \beta\right) \cos \beta \sec \theta_{s}$

which defines the first bubble's $B_{1}$ internal energy $U_{1}$ to

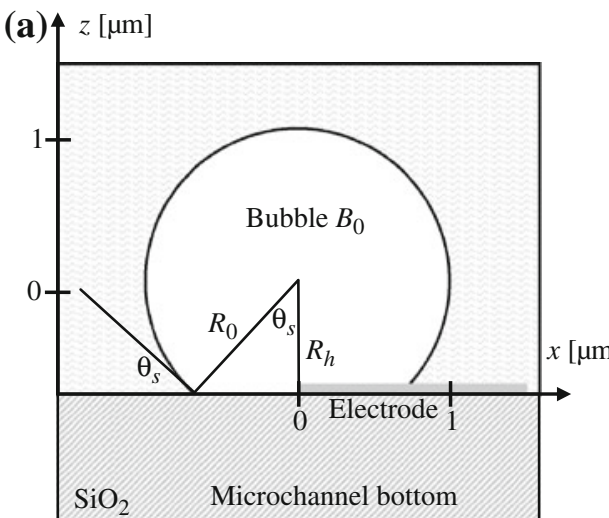

Fig. 2 Sketch of the spherical initial bubble $B_{0}$. a Vertical crosssection through bubble center revealing the height of the bubble center $R_{h}$. b Horizontal cross-section through bubble center revealing (b)

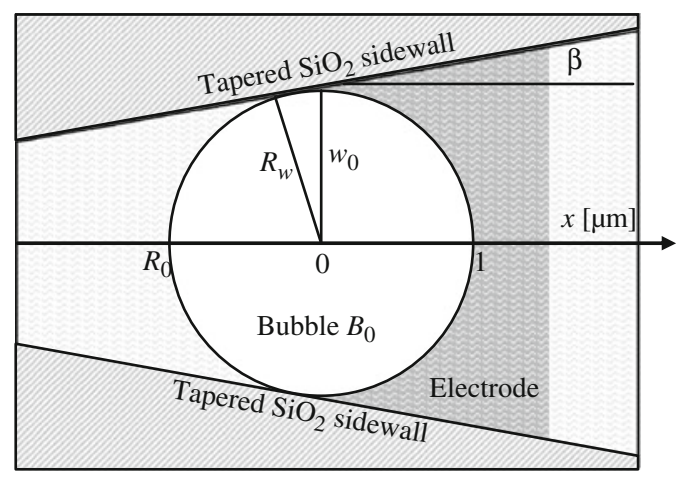

the maximum radius of the initial bubble $R_{0}=R_{w}$. In this sketch, the initial opening $w_{0}=1 \mu \mathrm{m}$, the taper angle $\beta=10^{\circ}$ and the static contact angle $\theta_{s}=45^{\circ}$ 
(a)

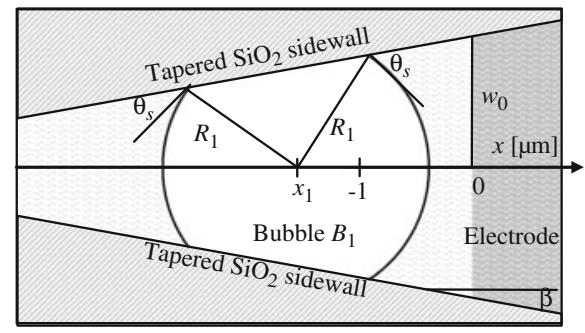

Fig. 3 a Horizontal cross-section of the first bubble $B_{1}$ through its center. It reveals the new bubble radius $R_{1}$ and its position within the microchannel $x_{1}$. In this sketch, the initial opening $w_{0}=1 \mu \mathrm{m}$, the taper angle $\beta=10^{\circ}$ and the static contact angle $\theta=45^{\circ}$. b On the left

$$
\begin{array}{r}
U_{1}=\frac{\gamma_{l a}}{R_{1}}\left(\int_{-R_{1}}^{R_{1} \cos \theta_{s}} \int_{0}^{2 \pi} \int_{0}^{\sqrt{R_{0}^{2}-z^{2}}} r \mathrm{~d} r \mathrm{~d} \omega \mathrm{d} z\right. \\
\left.-2 \int_{R_{1} \cos \theta_{s}}^{R_{1}} \int_{0}^{2 \pi} \int_{0}^{\sqrt{R_{0}^{2}-z^{2}}} r \mathrm{~d} r \mathrm{~d} \omega \mathrm{d} z\right) .
\end{array}
$$

This transition from the initial bubble $B_{0}$ to the first bubble $B_{1}$ is considered to be isothermal. Moreover, the bubble positions and deforms itself faster than new gas is generated by electrolysis. The rate of the gas development can be adjusted by the applied current and this can be chosen small enough to fulfill this criteria, and hence, the bubble can be considered as a closed system during this short transition phase. According to the ideal gas law, the internal energy $U_{\text {int }}$ then remains constant

$U_{\text {int }}=U_{0}=U_{1}$.

Inserting Eqs. 3 and 5 into Eq. 6, replacing the radius $R_{1}$ with Eq. 4, and solving of the first bubble's center position $x=x_{1}$ yields

$x_{1}=w_{0} \cot \beta\left(\sqrt{\frac{\left(\cos \frac{\theta_{s}}{2}\right)^{4}\left(\cos \theta_{s}-2\right)}{3 \cos \theta_{s}+\sec \theta_{s}\left(2 \sec \theta_{s}-9\right)}}-1\right)$

Since the square root is less than 1 for $0<\theta_{s}<\pi / 2$, the bubble clearly detaches from the electrode and jumps into its new position $x_{1}<0$. This is illustrated in Fig. 3a where a horizontal cross-section through the center of the first bubble $B_{1}$ is shown. This shift in position needs to be considered for the placement of the electrode within the lgseparator. The bubble may not escape through the inlet of the lg-separator into the eo pump. The following effects may influence this movement: pinning of the gas bubble, as experienced in the work by Paust et al. (2009), might delay the positioning of the bubble from its initial position. Such

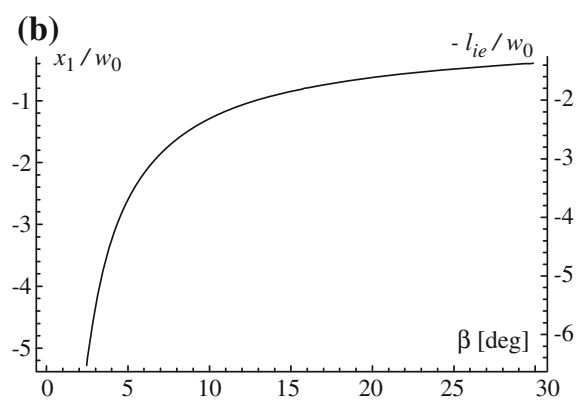

ordinate the position $x_{1}$ of the first bubble $B_{1}$ and on the right ordinate the minimum distance from the electrode to the inlet of the $\mathrm{lg}$ separator is shown as a function of the taper angle $\beta$

delay results in a larger bubble volume which leads to a shorter leap into the microchannel constriction. The same accounts for the contact angle hysteresis: the lower the receding contact angle $\theta_{r}$ and the larger the advancing contact angle $\theta_{a}$, the shorter the leap into the microchannel constriction. Within the experiments, no delay or complete pinning could be observed, the bubble detached smoothly from the electrode. The contact angle hysteresis was observed and measured as indicated above $\left(\theta_{r}=42^{\circ}\right.$, $\theta_{a}=47^{\circ}, \theta_{s}=45^{\circ}$ ). Therefore, we selected to work with the assumption of static conditions which represents a worst case scenario.

The position of the first bubble's center $x_{1}$ as a function of the taper angle $\beta$ is shown in Fig. 3b. It can be seen that for small taper angles $\beta$ the position $x_{1}$ of the first bubble reaches far into the narrow part of the microchannel. The maximum distance of the bubble's liquid-gas interface into the lg-separator is the same as the minimum distance $l_{i e}$ from the inlet of the lg-separator to the electrode. By the geometry, the minimum distance $l_{i e}$ can be calculated to

$$
\begin{aligned}
l_{i e}>w_{0} & \left(\cot \beta+\left(\cos \beta \sec \theta_{s}-\cot \beta\right)\right. \\
& \left.\times \sqrt{\frac{\left(\cos \frac{\theta_{s}}{2}\right)^{4}\left(\cos \theta_{s}-2\right)}{3 \cos \theta_{s}+\sec \theta_{s}\left(2 \sec \theta_{s}-9\right)}}\right)
\end{aligned}
$$

The minimum distance $l_{i e}$ to the inlet of the lg-separator is shown Fig. 3b, on the right ordinate axis.

\subsubsection{Bubble movement within the liquid-gas-separator}

During the electrolysis, continuously more bubbles start to grow at the electrode. These bubbles merge with the first bubble $B_{1}$, once their liquid-gas interfaces touch each other. This lets the first bubble $B_{1}$ grow and move towards the electrode and then past the electrode and finally out of the lg-separator. During this movement, it is important that the bubble does not disconnect or strongly constrain 
the electrical path between the electrodes, which would stop the eo pumping. This might occur in two different ways. First, the bubble may cover the electrode completely, hence, a minimum electrode length $l_{e}$ is required for preventing this, and second, the bubble stretches out over the complete cross-section and reaches the top, hence, a minimum height $h_{c}$ of the microchannel is required. Figure 4 shows the situation of the critical bubble $B_{c}$, which occurs once the bubble's smaller liquid air meniscus just touches the small side of the electrode on the microchannel bottom. The liquid-gas interface has a circular shape of $R_{b}$ at the bottom of the microchannel. By geometry, $R_{b}=w_{0} /$ $\cos \left(\theta_{s}+\beta\right)$, and the minimum electrode length $l_{e}$ equates to $l_{e}>2 R_{\mathrm{b}} \sin \left(\beta+\theta_{s}\right)=2 w_{0} \tan \left(\beta+\theta_{s}\right)$.

For the minimum height $h_{c}$, the radius $R_{c}$ of the critical bubble is needed and can be determined by geometrical considerations to $R_{c}=R_{b} / \sin \theta_{s}$. From this, the minimum height of the microchannel is

$h_{c}=R_{c}\left(1+\cos \theta_{s}\right)=w_{0} \sec \left(\theta_{s}+\beta\right)\left[\cot \theta_{s}+\csc \theta_{s}\right]$

The minimum electrode length $l_{e}$ and the minimum microchannel height $h_{c}$ as a function of the taper angle $\beta$ is shown Fig. 5. It can be seen that large taper angles $\beta$

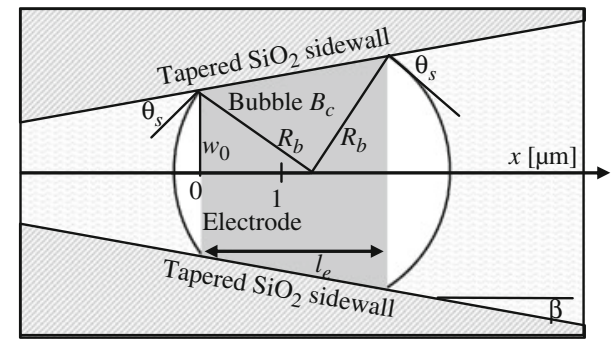

Fig. 4 Sketch of the critical bubble at the bottom of the microchannel. From this sketch the minimum electrode length $l_{e}$ can be determined. In this sketch, the initial opening $w_{0}=1 \mu \mathrm{m}$, the taper angle $\beta=10^{\circ}$ and the static contact angle $\theta_{s}=45^{\circ}$

(a)

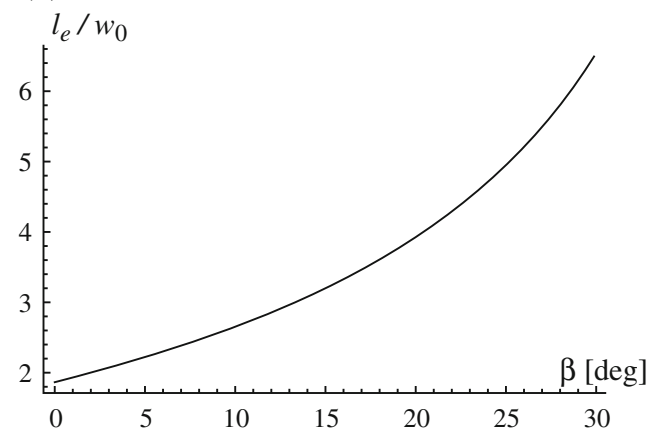

enforce an even larger electrode length $l_{e}$ and a larger minimum microchannel height $h_{c}$.

\subsubsection{Backpressure of the liquid-gas-separator}

During further actuation, the bubble in the outlet section of the lg-separator grows further and eventually stretches out over the complete cross-section. At this moment, the bubble separates the water column inside the microchannel into two detached liquid-gas interfaces, i.e., into two different menisci. Any pressure difference between the inlet and the outlet of the lg-separator acts directly on the bubble and displaces its respective menisci. In order to still reliably couple the current into the solution, the bubble may not be driven back over or behind the electrode, which could happen if the pressure on the meniscus facing the outlet exceeds the one acting on the other meniscus from the other side by a threshold value. This threshold is built up by the Young-Laplace pressure of the two separate menisci, and it can be considered as the backpressure tolerance $p_{b}$ of the lg-separator. In order to calculate this backpressure $p_{b}$, it is assumed that the microchannel is much higher than wide and, hence, the curvature of the meniscus is completely defined by the width (Han et al. 2006) which reduces the model to two dimensions. In this first assumption, also any shape deformation of the meniscus's liquid-gas interfaces by the rectangular corners, connecting the microchannel sidewall to the top and the bottom, respectively, is neglected. The bubble can then be represented by the two separated menisci, as shown in Fig. 6. The dependency of the backpressure $p_{b}$ on the initial microchannel width $w_{0}$ and the taper angle $\beta$ are best explained by setting the initial opening $w_{0}$ at the position $x_{1}$ of the first meniscus and define the position of the second meniscus by the bubble length $l_{b}$. Since the bubble is pushed out of the capillary the contact angle hysteresis needs to be considered (Paust et al. 2009), this results in an advancing contact angle $\theta_{a}$ for the first meniscus and a

(b)

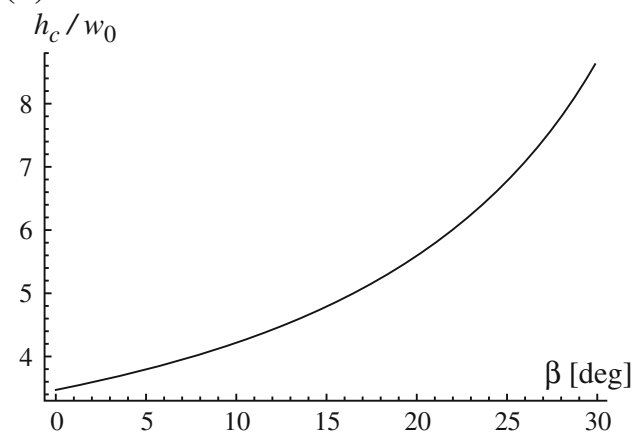

Fig. 5 a The dimensions of the critical bubble $B_{c}$ determine the minimum electrode length $l_{e}$ and the minimum microchannel height $h_{c}$. a The minimum electrode length $l_{e}$ and $\mathbf{b}$ the minimum microchannel height $h_{c}$ as a function of the taper angle $\beta$ 
receding contact angle $\theta_{r}$ for the second meniscus. The Young-Laplace pressure drop $p_{1}$ and $p_{2}$ over the respective menisci can be calculated to

$p_{1}=\frac{\gamma_{l a}}{R_{1}}=\gamma_{l a} \frac{\cos \left(\theta_{a}+\beta\right)}{w_{0}}$ and

$p_{2}=\frac{\gamma_{l a}}{R_{2}}=\gamma_{l a} \frac{\cos \left(\theta_{r}-\beta\right)}{w_{0}+l_{b} \tan \beta}$.

Furthermore, for the backpressure $p_{b}$ the bubble length $l_{b}$ is parameterized by the bubble parameter $b_{p}=l_{b} / w_{0}$ which leads to the backpressure as

$p_{b}=p_{1}+p_{2}=\frac{\gamma_{l a}}{w_{0}}\left[\cos \left(\theta_{a}+\beta\right)-\frac{\cos \left(\theta_{r}-\beta\right)}{1+b_{p} \tan \beta}\right]$.

As long as, $p_{b}$ is positive, the bubble will be reliably conducted away. In order to generate high backpressures $p_{b}$, the initial width $w_{0}$ of the microchannel at the electrode should be chosen as small as possible. The choice of the optimal taper angle $\beta$ is more complex. Figure 7 a shows the backpressure $p_{b}$ versus the parameterized bubble length $b_{p}$. For small parameterized bubble lengths $b_{p}$, the backpressure $p_{b}$ becomes negative and the bubble moves to lower values of $x$. For taper angles $\beta>\pi / 2-\theta_{a}$, the backpressure $p_{b}$

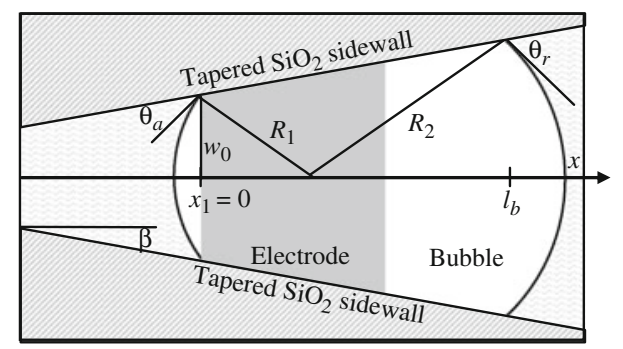

Fig. 6 Sketch of an horizontal cross-section showing the entrapped bubble stretched over the complete cross-section of the microchannel. The microchannel is cut along the symmetry line in its center. The sketch reveals the two menisci radii, $R_{1}$ and $R_{2}$ which are essential for the calculation of the lg-separator backpressure $p_{b}$

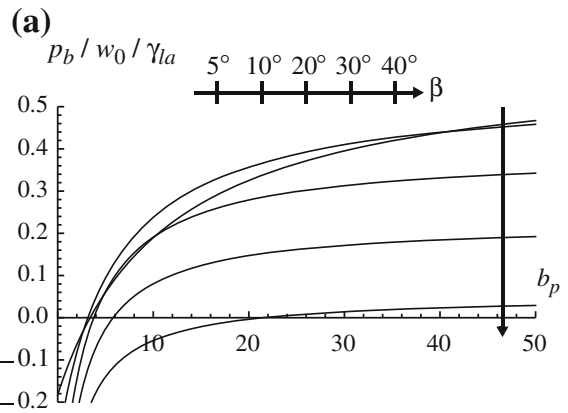

Fig. 7 a The lg-separator backpressure $p_{b}$ as a function of the bubble parameter. A minimum bubble length is required to have a positive backpressure $p_{b}$. At long bubble lengths the backpressure $p_{b}$ saturates to the Young-Laplace pressure of the first meniscus. The smaller the taper angle $\beta$, the higher the saturation backpressure $p_{b}$. b The always stays negative, which represents a hydrophobic behavior of the microchannel. The backpressure $p_{b}$ saturates for long bubbles in the Young-Laplace pressure $p_{1}$ of the first meniscus. The maximum bubble parameter $b_{p \max }$ is limited by the distance $l_{e o}$ of the electrode to the outlet of the lg-separator $b_{p \max }=l_{e o} / w_{0}$. Moreover, this assumes that the aspect ratio remains high towards the end of the lg-separator. The backpressure $p_{b}$ versus the taper angle $\beta$ is shown in Fig. $7 \mathrm{~b}$. The maximum backpressure $p_{b}$, reached by a specific taper angle $\beta$ can be determined from the graph. The backpressure $p_{b}$ raises steeply for small taper angles $\beta$ and declines slowly for larger taper angles $\beta$. The value of the taper angle $\beta$ for the maximum backpressure $p_{b}$ at a receding contact angle $\theta_{r}$ of $42^{\circ}$ and an advancing contact angle $\theta_{a}$ of $47^{\circ}$ stays below a taper angle $\beta$ of $12^{\circ}$ for all bubble lengths.

\subsubsection{Summary of the design parameters for the liquid-gas-separator}

Figure 8 shows an lg-separator with its design critical lengths. The microchannel width $w_{0}$ at the electrode and the taper angle $\beta$ should be chosen in order to guarantee a reproducible fabrication and a high backpressure $\mathrm{p}_{b}$ of the lg-separator to reliably conduct the electrolyzed gases away. From the above mentioned considerations, the smallest possible microchannel width $w_{0}$ should be chosen since all critical design values and the backpressure $p_{b}$ scale with the inverse of the width $w_{0}$. In addition a taper angle $\beta$ of $10^{\circ}$ represents a good choice. With this taper angle $\beta$, the electrode should be placed according to: the distance of the electrode to the inlet of the $\lg$-separator $l_{i e}$ is about $w_{0}$, see Eq. 8 and Fig. 3b. The minimum length of the electrode $l_{e}$ is about $4 w_{0}$, see Eq. 9 and Fig. 5a. The distance of the electrode to the outlet of the lg-separator $l_{e o}$ is about $20 w_{0}$, see Eq. 13 and Fig. 7. The microchannel should have a minimum height $h_{c}$ of about $5 w_{0}$, see Eq. 10 and Fig. $5 \mathrm{~b}$.

(b)

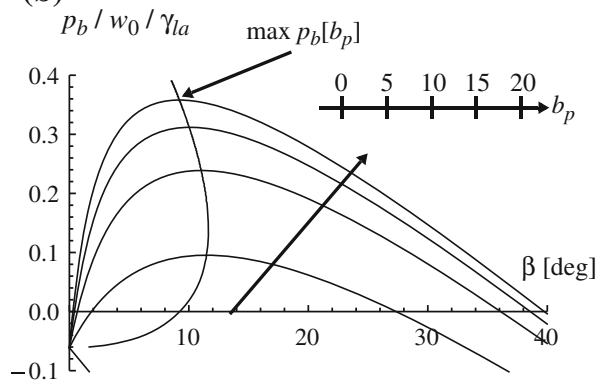

backpressure $p_{b}$ as a function of the taper angle $\beta$. At small taper angles $\beta$ the backpressure $p_{b}$ steeply increases until it reaches its maximum. This maximum is reached, for an receding contact angle $\theta_{r}=42^{\circ}$ and an advancing contact angle $\theta_{a}=47^{\circ}$ and for a taper angle $\beta<12^{\circ}$ for all bubble lengths 


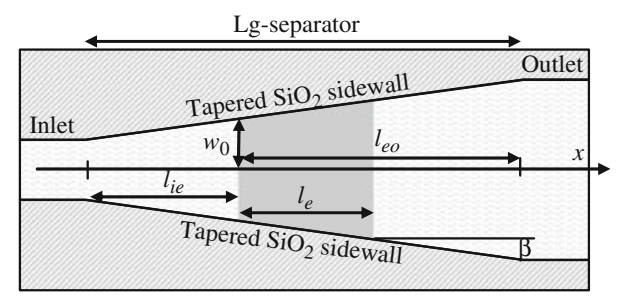

Fig. 8 Sketch of the $1 g$-separator showing its critical dimensions. The microchannel sidewall has the opening of $w_{0}$ at the electrode. The microchannel sidewall is tapered with the angle $\beta$. The electrode has the length $l_{e}$. The small electrode edge has a distance $l_{i e}$ from the inlet and a distance $l_{e o}$ from the outlet

\subsection{Model of the fluidic system with an embedded electroosmotic pump}

A sketch of a fluidic system with an embedded eo pump is shown in Fig. 9a. The chip is fluidically contacted via large reservoirs (capacity $0.2 \mu \mathrm{l}$ ). Within the chip, the eo pump is fluidically contacted by the pump inlet and pump outlet microchannel and electrically contacted via the electrodes placed inside an lg-separator.

\subsubsection{Filling of the fluidic system and operation of the electroosmotic pump}

In order to derive the boundary conditions for the modeling, the filling and the operation of the eo pump needs to be taken into account. For the experiment, a droplet of aqueous solution was placed in the reservoir of the pump inlet and outlet. The Pt electrodes were connected through the contact pads, which were situated in the opening of the exhaust microchannel. By applying a voltage between the two electrodes, a current was coupled into the solution. This started the electrolysis and the lg-separator conducted the emerging gas out of the exhaust microchannel. At the same time, the current coupled into the solution led to: a proportionally small voltage drop over the first electrode inside the lgseparator and the pump inlet, a proportionally larger voltage drop over the eo pump, and a proportionally small voltage drop from the pump outlet to the second electrode, in the second lg-separator. These voltage drops induced eo pressures and thereby induced a flow through the inlet microchannel towards the pump, as well as, a flow out of the pump into the outlet microchannel.

\subsubsection{Flow determination within the fluidic system}

Next to the eo induced flow the model needs to integrate the influence of evaporation driven flow through the exhaust microchannel, hydrostatic pressure induced flow, and flow induced due to misalignment of the electrodes

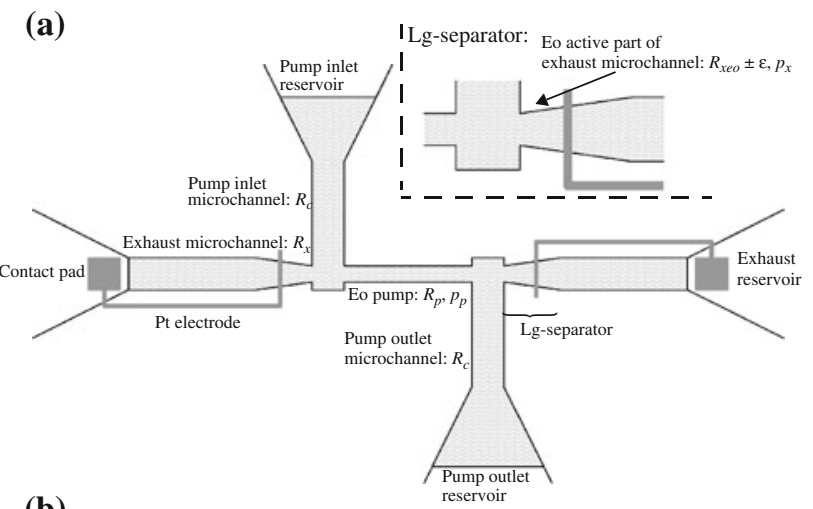

(b)

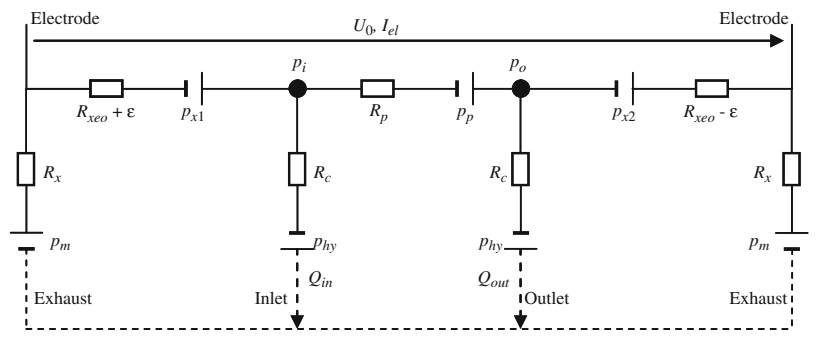

Fig. 9 a Schematic sketch of fluidic system including the eo pump with lg-separator. b Equivalent electrical circuit for the determination of flow and pressures within the fluidic system

inside the lg-separator. The layout of the eo pump, as shown in Fig. 9a, can be transformed into an equivalent electrical circuit, as shown in Fig. 9b. The flows and pressures within the eo pump can be determined by solving the equivalent electrical circuit diagram. The microchannels are replaced by resistors with a specific hydraulic resistance as described by Morf et al. (2001). $R_{c}, R_{p}, R_{x}$, denote the hydraulic resistance of the pump inlet and outlet, the eo pump, and the exhaust microchannel, respectively. The eo pressures are represented by voltage sources, $p_{p}$ and $p_{x}$ for the eo pump and the eo active part of the exhaust microchannel. This yields the following equations:

$p_{p}=\mu_{e o} \rho_{e l} R_{p} I_{e l} \quad$ and $\quad p_{x 1,2}=\mu_{e o} \rho_{e l}\left(R_{x e o} \pm \varepsilon\right) I_{e l}$,

where $R_{x e o}$ represents the average hydraulic resistance of the eo active part of the exhaust microchannel and $\varepsilon$ the difference in hydraulic resistance according to an alignment mismatch of the electrodes.

Furthermore, the hydrostatic pressure $p_{h y}$ could have been integrated into the model as an initially charged capacitor. Its value represents the different filling levels in the reservoir of the pump inlet and outlet, as well as, an inclination of the chip. However, the consideration as a time independent voltage source $p_{h y}$ simplifies the model and is a reasonable approximation since the displaced liquid volumes are small compared to the volume of the reservoir of about $0.2 \mu \mathrm{l}$. If, for example, both reservoirs are half filled, a displaced volume of $10 \mathrm{pl}$ results in a hydrostatic pressure change of $1 \mu \mathrm{Pa}$, which is negligible 
compared to the experienced pressures within the fluidic system.

At the transition between the exhaust microchannel and the reservoir, a meniscus is formed, due to the sudden enlargement of the cross-section. The Young-Laplace pressure of this meniscus prevents a filling of the exhaust reservoir through the microchannel. The pressure drop over the meniscus is by an additional voltage source $p_{m}$. Without any voltage applied, water already evaporates from the meniscus, inducing an evaporation driven flow $v$. This evaporation driven flow defines the value of the voltage source $p_{m}=v\left(R_{c}+R_{x}\right)$ at $I_{e l}=0$. Once the pump is actuated, the shape of the meniscus adjusts according to the applied pressure at the exhaust microchannel outlet, which slightly changes the evaporation area, and thus the evaporation induced flow $v$.

Integrating all these effects leads to a definition of the Kirchhoff equations for the independent pressure at the pump inlet $p_{i}$ and pump outlet $p_{o}$ as:

$$
\begin{aligned}
& \frac{p_{i}-p_{x 1}-p_{m}}{R_{x}}+\frac{p_{i}+p_{h y}}{R_{c}}+\frac{p_{i}-p_{o}+p_{p}}{R_{p}}=0 \\
& \frac{p_{o}-p_{x 2}-p_{m}}{R_{x}}+\frac{p_{o}-p_{h y}}{R_{c}}+\frac{p_{o}-p_{o}-p_{p}}{R_{p}}=0
\end{aligned}
$$

Solving this system of equations for $p_{i}$ and $p_{o}$ leads to the flow $Q_{\text {out }}$ into the outlet microchannel and the flow $Q_{\text {in }}$ into the inlet microchannel as:

$$
\begin{aligned}
Q_{\text {out }, \text { in }}= & v \pm \frac{p_{h y} R_{p} R_{x}}{R_{c}\left[R_{p} R_{x}+R_{c}\left(R_{p}+2 R_{x}\right)\right]} \\
& \pm \mu_{e o} \rho_{e l} I_{e l}\left(\frac{R_{p}\left(R_{x}-R_{x, i n c l}\right)}{R_{p} R_{x}+R_{c}\left(R_{p}+2 R_{x}\right)} \mp \frac{\varepsilon}{R_{c}+R_{x}}\right) .
\end{aligned}
$$

Measuring $Q_{\text {out }}$ and $Q_{\text {in }}$, and plotting it versus $I_{e l}$, reveals the contributions from the evaporation induced flow $v$, the hydrostatic pressure induced flow $p_{h y}$ and the flow induced by the misalignment of the electrodes $\varepsilon$. At $I_{e l}=0$ the evaporation induced flow $v$ can be seen as the average offset of $Q_{\text {out }}$ and $Q_{\text {in }}$ :

$\left.\frac{Q_{\text {out }}+Q_{\text {in }}}{2}\right|_{I_{e l}=0}=v$.

The hydrostatic pressure difference $p_{\text {hy }}$ can be determined from the difference between $Q_{\text {out }}$ and $Q_{\text {in }}$ :

$\left.\frac{Q_{\text {out }}-Q_{\text {in }}}{2}\right|_{I_{\text {el }}=0}=p_{h y} \frac{R_{p} R_{x}}{R_{c}\left[R_{p} R_{x}+R_{c}\left(R_{p}+2 R_{x}\right)\right]}$.

The misalignment of the electrodes is expressed in the derivative of the average of $Q_{\text {out }}$ and $Q_{i n}$ with respect to the current $I_{e l}$ :

$\frac{d}{d I_{e l}}\left(\frac{Q_{o u t}+Q_{\text {in }}}{2}\right)=\varepsilon \frac{\mu_{e o} \rho_{e l}}{R_{c}+R_{x}}$.

\subsubsection{Dimensioning of the microchannels}

The requirements on the design of the fluidic system were: a robust implementation regarding the manufacturing process and the actuation, and flows in the range of $10 \mathrm{pl} \mathrm{s}^{-1}$, which amounts to an average velocity of $50 \mu \mathrm{m} \mathrm{s}^{-1}$ inside the pump outlet microchannel. For a sufficiently high aspect ratio (height to width) within the eo pump and the lg-separator, the deep reactive ion etching (DRIE) of the microchannels' height was targeted to be $10 \mu \mathrm{m}$ in the widest microchannels. For the pump outlet and inlet, microchannels with a width of $20 \mu \mathrm{m}$ were chosen. This led to a hydraulic resistance for a $800 \mu \mathrm{m}$ long microchannel which was low compared to the hydraulic resistance of the eo pump. The eo pump had a width of $1.5 \mu \mathrm{m}$. This implied a trade-off between reliability during fabrication, appropriate pump backpressure $p_{b}$ and sensitivity towards clogging. The minimum length of the pump is determined by the rounding of the corners during lithography and thermal oxidation. The minimum pump length was set to $30 \mu \mathrm{m}$, for a welldefined hydraulic resistance of the pump. In order to increase the flow, two of those pumps were connected in parallel, which halved the hydraulic and electrical resistance. The exhaust microchannel's width was designed to $20 \mu \mathrm{m}$ and a length of $800 \mu \mathrm{m}$ to reach the reservoir. This exhaust microchannel was placed at the outlet of the $1 g$ separator. The design of the lg-separator was more complex. In order to have the highest backpressure $p_{b}$ capacity of the $1 \mathrm{~g}$-separator, an inlet width of $1.5 \mu \mathrm{m}$ was chosen. The taper angle $\beta$ was defined to reach a high backpressure $p_{b}$ while maintaining at the same time the ability to reliably be defined during fabrication. During mask fabrication a tapered line was represented by a pixelated step-like profile with a minimum step size of $250 \mathrm{~nm}$. These steps needed to be reliably smoothened out during lithography, DRIE and thermal oxidation. A safe choice seemed to be a taper angle of $\beta=11^{\circ}$, resulting in 39 steps over a length of $50 \mu \mathrm{m}$. In order to increase the reliability of $1 g$-separator and to reduce the influence of the corner rounding, a $5 \mu \mathrm{m}$ long microchannel was placed between the entrance of the lg-separator and the eo pump. Finally, the electrode was placed at a distance of $10 \mu \mathrm{m}$ from the inlet of the lg-separator. This provided enough margin for a possible electrode misalignment. All this resulted in a complete footprint of 100 $\mu \mathrm{m} \times 15 \mu \mathrm{m}$ (length $\times$ width) of the eo pump.

\section{Fabrication}

\subsection{Process flow}

For the microfluidic system, microchannels with a constant height and variable width, and fluidic connections were 
(a)

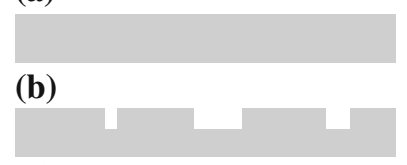

(c)

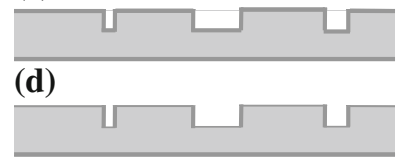

(e)

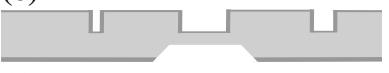

(f)

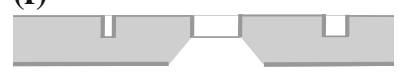

Fig. 10 Process flow for the integration of Pt electrodes into the microfluidic microchannels. a Double sided polished $525 \mu \mathrm{m}$ wafer. b Outlining the microchannels as DRIE trenches. c Thermal oxidation in order to narrow down the width of the microchannels. d RIE to thin down the thermal oxide layer on the front surface for reliable bonding. e $\mathrm{KOH}$ etching the reservoirs as cavities from the backside of the wafer, stopping inside the Si. f Final etching of the cavities in TMAH for soft landing in $\mathrm{SiO}_{2}$ membrane. $\mathrm{g}$ RIE from the backside to remove the $\mathrm{SiO}_{2}$ for good electrical contact during anodic bonding and to remove the $\mathrm{SiO}_{2}$ membrane inside the cavities for fluidic connection. $\mathbf{h}$ Boron type glass wafer for anodic bonding. i Lift-off metallization of $15 \mathrm{~nm}$ Ta and additional $150 \mathrm{~nm} \mathrm{Pt}$. j Anodic bonding of the two wafers to close the microchannels

required, as well as, electrodes inside the microchannels for eo, on-chip pumping. The process flow was based on photolithography, Si etching, thermal oxidation, lift-off metallization and anodic bonding for a two wafer process. A simplified process flow is shown in Fig. 10. (a) The first wafer was a double-side polished, $525 \mu \mathrm{m}$ thick silicon $(\mathrm{Si})$ wafer. (b) The microchannels were outlined by DRIE into the frontside of the wafer. The trench-width varied from $2 \mu \mathrm{m}$ up to $20 \mu \mathrm{m}$ with a depth of $10 \mu \mathrm{m}$ for the $20 \mu \mathrm{m}$ wide microchannel. (c) These microchannels were subsequently narrowed by thermally growing $1.5 \mu \mathrm{m}$ of $\mathrm{SiO}_{2}$, which reduced the initial width by $1.65 \mu \mathrm{m}(55 \%$ of the $\mathrm{SiO}_{2}$ grows out of the $\mathrm{Si}$ surface; Madou 2002). (d) As it turned out, $\mathrm{a} \mathrm{SiO}_{2}$ thickness of $1.5 \mu \mathrm{m}$ was too thick for a reliable anodic bond, because of an insufficiently strong electrical field between the two wafers. Consequently, the $\mathrm{SiO}_{2}$ surface was highly anisotropically thinned by reactive ion etching (RIE) to a final thickness of $100 \mathrm{~nm}$, as suggested by Lee et al. (2000) and Plaza et al. (1998). Advantageous was also that more ions were implanted into the $\mathrm{SiO}_{2}$, which further enhanced the bonding strength. (e) The fluidic connection to the chip was established by $\mathrm{Si}$-wet-etching the reservoirs. These reservoirs were shaped as cavities through the wafer to also fluidically connect the backside to the frontside. The etching was performed from the backside through the wafer and stopped at the front side at the thin $\mathrm{SiO}_{2}$ membrane. The $\mathrm{Si}$-wet-etching was first performed in potassium hydroxide $(\mathrm{KOH})$ for a high etch rate and (f) then finished in tetramethylammonium hydroxide (TMAH) for a gentle stop at the $\mathrm{SiO}_{2}$. This protected the $\mathrm{SiO}_{2}$ frontside from being etched, in case a membrane broke. (g) Finally, the complete $\mathrm{SiO}_{2}$ was removed from the backside by RIE to ensure a good electrical contact and to open the membranes for the fluidic connection. (h) The second wafer was a standard boron type glass wafer for anodic bonding. (i) The metallization of $15 \mathrm{~nm}$ tantalum (Ta) and $150 \mathrm{~nm}$ Pt was deposited in a lift-off process. (j) Both wafers were $\mathrm{RCA}^{1}$ cleaned right before the anodic bonding process. The wafers were aligned in such a way that the microchannels ended in the fluidic connection. The wafers were anodically bonded at $400^{\circ} \mathrm{C}$ and an applied voltage of $1000 \mathrm{~V}$ for $1.5 \mathrm{~h}$.

\subsection{Fabrication results and discussion}

The microchannel's dimensions were measured for accurately modeling the device. The first measurement determined the height of the microchannels. The well-known phenomena of an aspect ratio dependent etching for DRIE was investigated, the wider the opening, the faster the etch process (Madou 2002). It was necessary to extract the precise height of the microchannels with different widths from the test run. Figure 11a shows a cross-section of DRIE trenches with a width from 1 to $20 \mu \mathrm{m}$. $50 \mu \mathrm{m}$ deep trenches were etched, where the etch depth was adjusted for the widest microchannel. Figure $11 \mathrm{~b}$ shows the normalized etch depth with regard to the $20 \mu \mathrm{m}$ wide microchannel. This can be used to determine the different microchannel heights of the fluidic system and, hence, their hydraulic resistance. Figure 12a shows two important results: the width of the eo pump and the rounding of the corners. The width of the eo pump can be determined to $1.5 \mu \mathrm{m}$. The intended width of $0.5 \mu \mathrm{m}$ could not be reached probably due to widening during the DRIE. In future, this needs to be narrowed down in order to generate higher eo pump backpressures. The corners were initially rounded with a radius of $2 \mu \mathrm{m}$ in the mask design to avoid stress concentrations and ensure repeatability in fabrication. The corner radius increased only up to $2.2 \mu \mathrm{m}$ during fabrication. The cross-section of the microchannel was investigated by cutting it open, as shown in Fig. 12b. For a $20 \mu \mathrm{m}$ deep and $4 \mu \mathrm{m}$ wide microchannel, an inclination angle of the sidewall of $89^{\circ}$ was measured, justifying the assumption of a rectangular microchannel cross-section. In addition, the $1.4 \mu \mathrm{m}$ thick $\mathrm{SiO}_{2}$ microchannel sidewall can be seen, indicated by the higher greyscale. Finally, the top corner of the microchannel rounded off during DRIE,

$\overline{{ }^{1} \mathrm{NH}_{4} \mathrm{OH}: \mathrm{H}_{2}} \mathrm{O}_{2}: \mathrm{H}_{2} \mathrm{O}=1: 1: 5$ at $70^{\circ} \mathrm{C}$ for $1 \mathrm{~h}$. 
(a)

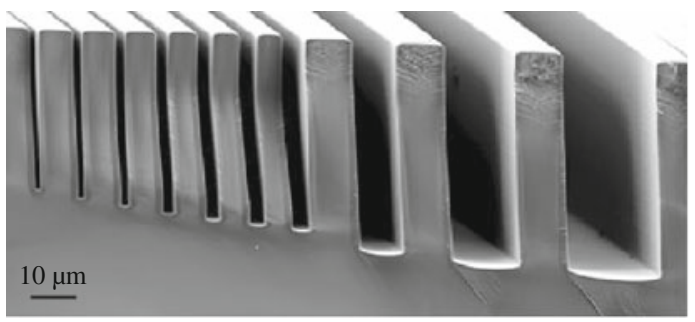

(b)

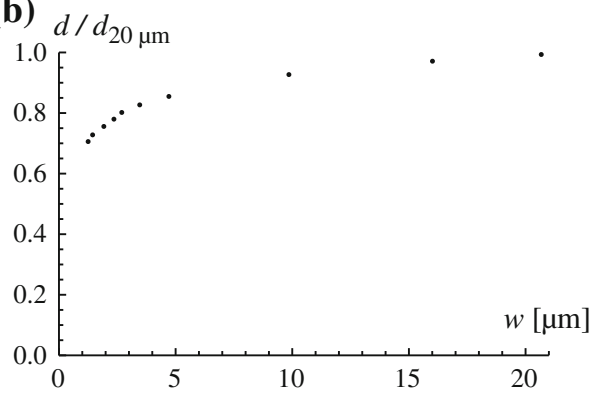

Fig. 11 a Scanning electron microscope (SEM) image showing that the depth of the DRIE trenches depended on the width of the microchannels. b The normalized depth with respect to a $20 \mu \mathrm{m}$ wide microchannel, extracted from a

(a)

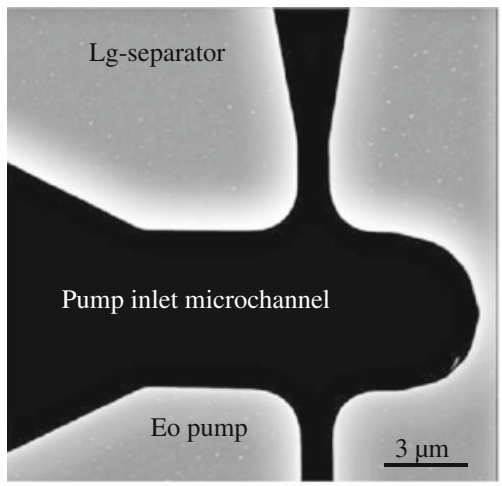

(b)

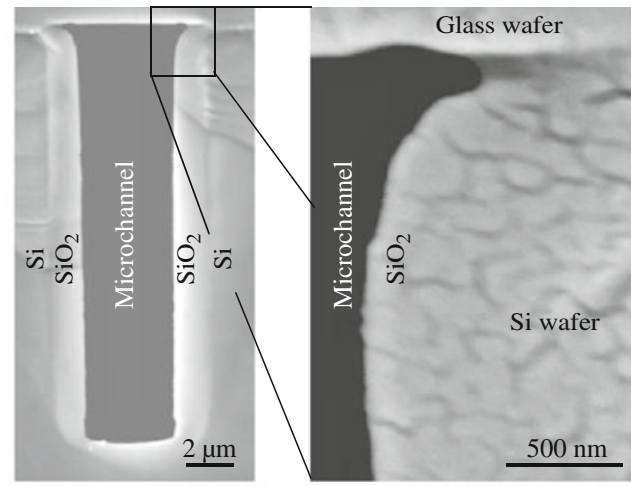

(d)

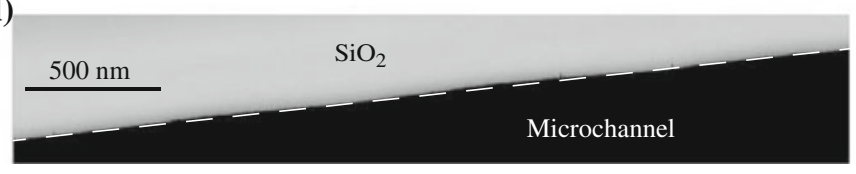

Fig. 12 SEM images of: a Top view of a DRIE and thermally oxidized pump inlet with the exhaust microchannel on the top and part of the eo pump at the bottom. The corners were rounded with a radius of $2.2 \mu \mathrm{m}$. The width of the eo pump was determined to $1.5 \mu \mathrm{m}$. b, $\mathbf{c}$ Shows a cross-section of pumping microchannel. $\mathbf{b}$ The sidewall of the microchannel deviated of about $1^{\circ}$ from verticality.
The thermal oxide thickness can be determined to $1.4 \mu \mathrm{m}$. c The imperfection of the bonding on the top corner resulted in enhanced corner flow but has no major consequences during the actuation of the pump. $\mathbf{d}$ The stepping of the tapered sidewall of $5^{\circ}$ resulted in a local variation of the taper angle $\beta \pm 0.6^{\circ}$ after etching and thermal oxidation thermal oxidation and RIE. The radius for this edge was measured to be $0.6 \mu \mathrm{m}$. After bonding, this recess resulted in a shallow $0.3 \mu \mathrm{m}$ wide and $0.2 \mu \mathrm{m}$ high additional microchannel, see Fig. 12c. This shallow part of the microchannel did not make a contribution to the hydraulic resistance but increased the cornerflow. A closer look was taken at the pixelation of the tapered sidewall to determine the minimum taper with acceptably rounded off steps. The problem with the pixelation of diagonal lines in the mask design is addressed in Fig. 12d. For a low taper angle $\beta$ of $5^{\circ}$, only a slight deviation from the diagonal line of $20 \mathrm{~nm}$ per $1 \mu \mathrm{m}$ long step was detected. This resulted in a local variation of the taper angle $\beta$ of $\pm 0.6^{\circ}$.

A result of the Pt lift-off process is shown in Fig. 13a. A triangle with an opening angle of $25^{\circ}$ resulted in a curved edge with a radius of $500 \mathrm{~nm}$. This was a sufficiently small feature size, since the minimum Pt features in this design were $10 \mu \mathrm{m}$. However, the $185 \mathrm{~nm}$ thick Ta/Pt line had a strong influence on the bonding quality, as shown in Fig. 13b. It resulted in a non bonded area along the Pt line with a distance up to $25 \mu \mathrm{m}$ away from the Pt edge. This non bonded area decreased the closer it reached the microchannel. Two effects can explain this void in the bond: the steric hindrance of the Pt line and the gas enclosure during the bonding step underneath the electrode. These gases, emerging during the bonding, were partially removed via the microchannels, which were connected to the outside. Applying a vacuum, in the range of $10 \mathrm{mbar}$, to support the extraction of this gas, did not increase the bonding quality. In this case, a plasma occurred between 
(a)

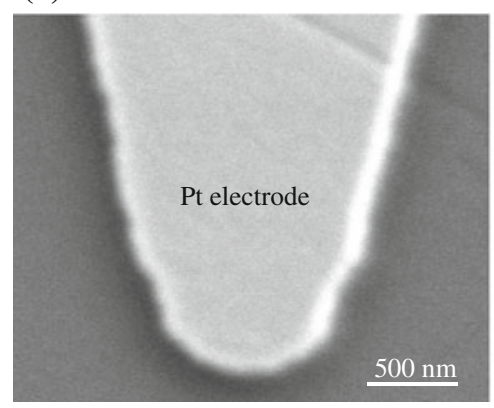

(b)

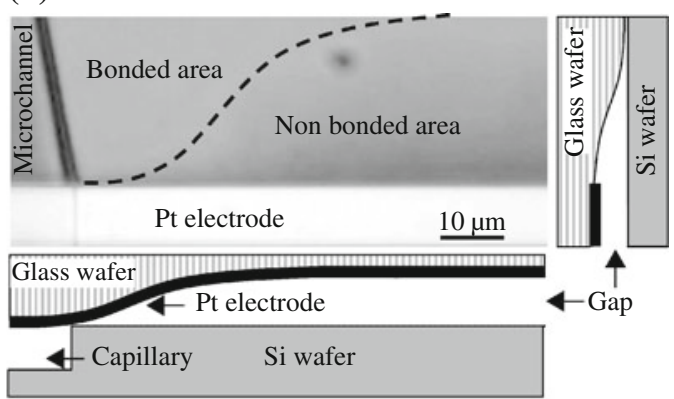

Fig. 13 a SEM image of a Pt lift-off fabricated tip with an opening angle of $25^{\circ}$. The tip rounded of with a radius of $500 \mathrm{~nm}$, which defines a sufficient small feature size for the fabrication. b Optical microscope image of a non bonded area underneath the Pt electrode.
This imperfection imposed a major reliability issue during actuation since hydrolysis can occur in this small gap and the emerging gases may leak into the pumping microchannel

Table 1 Dimensions fluidic system

\begin{tabular}{|c|c|c|c|c|c|c|}
\hline Pump inlet/outlet microchannel & Height & $10 \mu \mathrm{m}$ & Width & $20 \mu \mathrm{m}$ & Length & $800 \mu \mathrm{m}$ \\
\hline $\begin{array}{l}\text { EO pump, two parallel } \\
\text { microchannels }\end{array}$ & Height & $7.5 \mu \mathrm{m}$ & Width & $1.5 \mu \mathrm{m}$ & Length & $30 \mu \mathrm{m}$ \\
\hline Lg-separator & Average height & $8.8 \mu \mathrm{m}$ & Inlet width/taper angle & $1.5 \mu \mathrm{m} / 11^{\circ}$ & $\begin{array}{l}\text { Total length/electrode } \\
\text { placement }\end{array}$ & $50 \mu \mathrm{m} / 10 \mu \mathrm{m}$ \\
\hline Exhaust microchannel & Height & $10 \mu \mathrm{m}$ & Width & $20 \mu \mathrm{m}$ & Length & $800 \mu \mathrm{m}$ \\
\hline
\end{tabular}

the electrodes where the plasma current limited the applied voltage of the bonding setup to less than $1000 \mathrm{~V}$. These gaps imposed a major drawback, not because of a liquid connection, since their hydraulic resistance was comparably high, but because hydrolysis occurred at higher currents in these gaps. These emerging electrolyzed gases escaped into the pumping microchannel. The microchannel dimensions after fabrication are summarized in Table 1.

\section{Experiments, results, and discussion}

In the first part of this section, the lg-separator for reliably venting the electrolyzed gases was investigated with respect to its backpressure $p_{b}$ tolerance and its gas flow driving capability. In the second part, the eo pump was analyzed regarding to its linearity of the pump rate versus the electrical current, and the validity of the fluidic systems model. For each experiment, the chips were cleaned in an oxygen plasma for $20 \mathrm{~min}$ to remove any organic residues and to activate the $\mathrm{SiO}_{2}$ surface. For the experiments the microchannels were filled with deionized water ${ }^{2}$ having a specific resistivity of $12 \mathrm{M} \Omega \mathrm{cm}$. As flow marker a low concentration of $0.1 \mathrm{mM}$ fluorescein ${ }^{3}$ solution was employed.

\footnotetext{
2 Elga, Purelab UHQ.

${ }^{3}$ Fluka, Fluorescein sodium.
}

\subsection{Liquid-gas-separator}

The first experiment verified Eq. 11, which states that the Young-Laplace pressure depends on the taper angle $\beta$. The experiment compared two taper angles $\beta_{1}, \beta_{2}$ by passively entrapping a gas bubble in a $10 \mu \mathrm{m}$ deep microchannel with a maximum width at the meniscus of $5 \mu \mathrm{m}$. An optical image of the microchannel layout is shown in Fig. 14a. The microchannels were filled by water flowing in from the bottom and the air being pushed out of the system through the top. The flow split at the split-point into two independent flows. In the following section of the microchannel, it was important that the meniscus progressed with the same speed, until it reached the entrapment-point. From that moment onwards, the gas bubble was entrapped in the middle microchannel and compressed by the YoungLaplace pressure. After a certain time the bubble stayed at its equilibrium position, hence, the pressure inside the gas bubble was constant and must have matched the YoungLaplace pressure of the two entrapping menisci.

For a set of paired taper angles $\beta_{1}, \beta_{2}$ two different meniscus positions were investigated by entrapping two different volumes of air. For the analysis, the microchannels were filled and the images were taken immediately afterwards, in order to avoid the dissolution of air in water. According to Eq. 11, the Young-Laplace pressure was equal for both menisci in case their radius was equal. For the investigated taper angles $\beta$ a good matching of the 
(a)

(c)
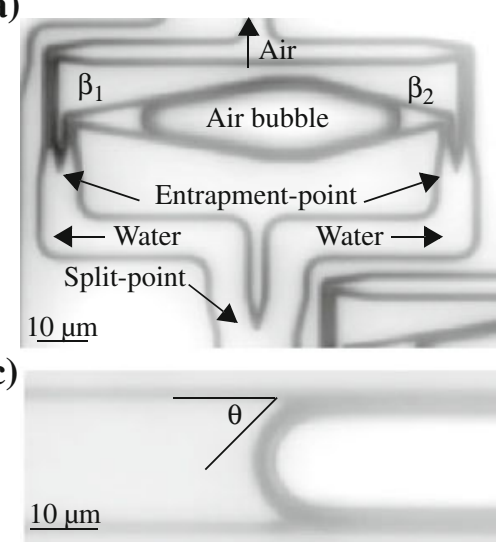

(b)

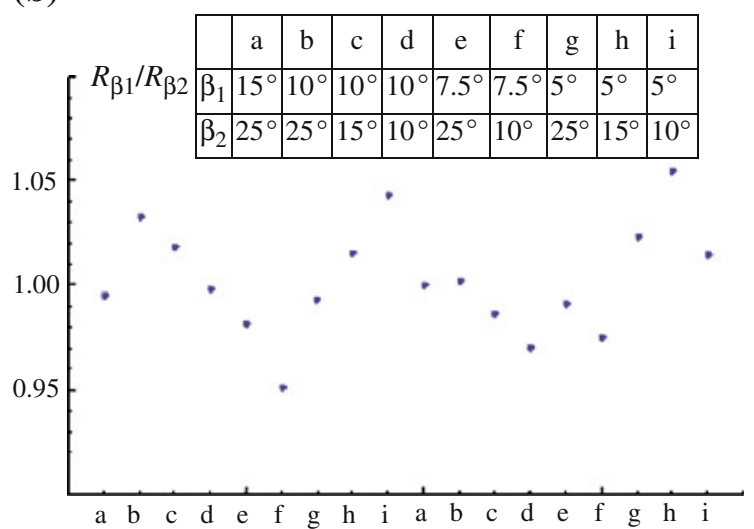

Fig. 14 a Optical microscope image of an entrapped air bubble in tapered microchannel. b Ratio between the radii of two opposing meniscus in a microchannel with two different taper angles $\beta_{1}$ and $\beta_{2}$.
Optical microscope topview image of the static contact angle $\theta_{s}=45^{\circ}$ inside the microchannel

separator can be calculated to $p_{b}=7 \mathrm{kPa}$. This defined its tolerance towards pressure changes which can be caused by contact angle changes due to contamination or cross-section changes.

Finally, the gas flow driving capability of the lg-separator integrated into the eo pump and the contact angle hysteresis within the exhaust microchannel was investigated, at a driving current of $20 \mathrm{nA}$ and with using deionized water. Figure 15 shows three images, taken with a time difference of $7 \mathrm{~s}$, of the emerging gas bubbles in the exhaust microchannel. Based on that, the flow of hydrolyzed gas was evaluated to be $2 \mathrm{pl} \mathrm{s}^{-1}$. From 13 bubbles seen in these images, the receding contact angle $\theta_{r}=42^{\circ} \pm 3^{\circ}$ and advancing contact angle $\theta_{a}=47^{\circ} \pm 4^{\circ}$ of water on $\mathrm{SiO}_{2}$ was determined in a similar way as described above for the static contact angle $\theta_{s}$.

\subsection{Electroosmotic flow determination}

\subsubsection{Experimental setup}

For the eo induced flow determination, deionized water as the propelled liquid was chosen. Using deionized water resulted in a long Debye layer thickness $\lambda_{D}$ on the $\mathrm{SiO}_{2}$ surface, due to its low concentration of ions, see also Bruus (2008) for more information. This led to a high eo mobility $\mu_{e o}$ and, hence, to an enhanced flow. A drawback of the current design was that water evaporated out of the pumping microchannel's reservoirs during the experiment and, therefore, keeping a constant concentration of ions in the solution was impossible. The evaporation would have also concentrated any contaminations in the reservoir and the microchannels. In order to perform several measurements with the eo pump, the least contaminating solution, deionized water, was chosen. To trace the pumping action 


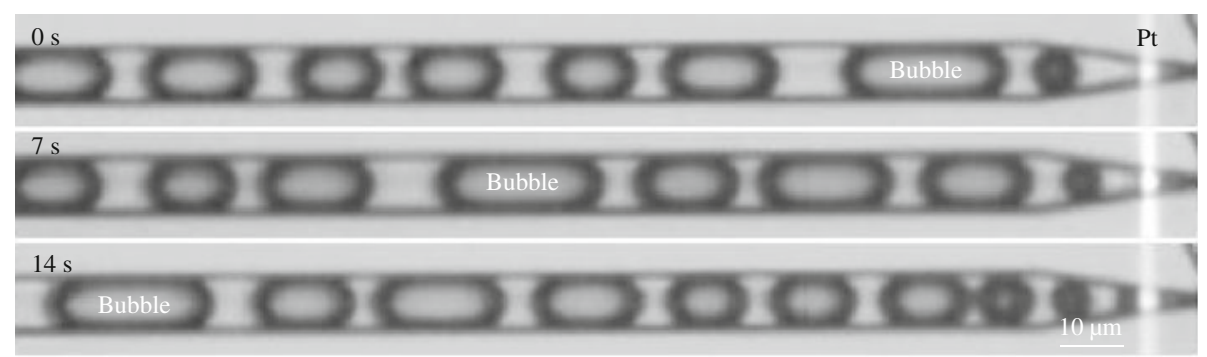

Fig. 15 Optical microscope image of an active lg-separator with a driving current of $20 \mathrm{nA}$. The time difference between the images was $7 \mathrm{~s}$. The hydrogen evolution is estimated to generate a flow of
$2 \mathrm{pl} \mathrm{s}^{-1}$. In addition, the receding contact angle $\theta_{r}=42^{\circ} \pm 3^{\circ}$ and advancing contact angle $\theta_{a}=47^{\circ} \pm 4^{\circ}$ could be determined

solution were assumed to be more or less the same at the low concentration used. Hence, a greyscale of 0.35 was not affected by diffusion and its progression in the center of the microchannel was considered as the location of the original interface and traced. Figure 16 shows a series of five consecutive greyscale distributions along the microchannel center. From this, the velocity $v_{t p}$ of the transition point was obtained, which was then further transformed into the flow $Q$.

According to Bruus (2008), for a Poiseuille flow in a rectangular microchannel the velocity $v_{t p}$ in its center can be described as:

$$
\begin{aligned}
v_{t p} & =\frac{4 h^{2} \Delta p}{\pi^{3} \eta L} \sum_{n, \text { odd }}^{\infty} \frac{1}{n^{3}}\left[1-\frac{1}{\cosh \left(n \pi \frac{w}{2 h}\right)}\right] \sin \left(\frac{n \pi}{2}\right) \\
& =\frac{48 Q}{\pi^{3}\left(h w-0.63 h^{3}\right)} \sum_{n, \text { odd }}^{\infty} \frac{1}{n^{3}}\left[1-\frac{1}{\cosh \left(n \pi \frac{w}{2 h}\right)}\right] \sin \left(\frac{n \pi}{2}\right),
\end{aligned}
$$

where the hydrostatic pressure $\Delta p$ was replaced by $\Delta p=R_{h y} Q$ and the hydraulic resistance $R_{h y}$ was calculated as $R_{h y}=12 \eta L /\left(h^{3} w-0.63 h^{4}\right)$. Solving the sum numerically for the exhaust microchannel crosssection with $w=2 h$ yields the flow $Q$ depending on the center velocity $v_{t p}$ as:

$Q=\frac{h^{2} v_{t p}}{0.9974}$. of the pump outlet. A sequence of images was taken, as shown in Fig. 16, and analyzed according to the greyscale distribution in the center of the microchannel. A reference point, which was not affected by diffusion, needed to be found in order to follow the initial transition point between deionized water and fluorescein solution. Hence, it was assumed that the greyscale average between 0.55 and 0.15 represented half of the fluorescein concentration. In addition, the diffusion of fluorescein into deionized water and the diffusion of deionized water into the fluorescein

\footnotetext{
${ }^{4}$ Zeiss, Axiovert S40 with a mounted camera AxioCam Mrm and a metal halide lamp HXP 120.
}

\subsubsection{Flow measurement and model verification}

This subsection determines and compares the four flow contributions with the model: evaporation induced flow $v$, hydrostatic pressure $p_{h y}$ induced flow, eo flow induced by the misalignment of the electrodes $\varepsilon$, and the intended eo flow. For the model validation, the solution's specific resistivity $\rho_{e l}$ and the eo mobility $\mu_{e o}$ needed to be experimentally defined. The solution's resistance was determined from Fig. 17a showing the current versus the potential applied between the bondpads 
(a)

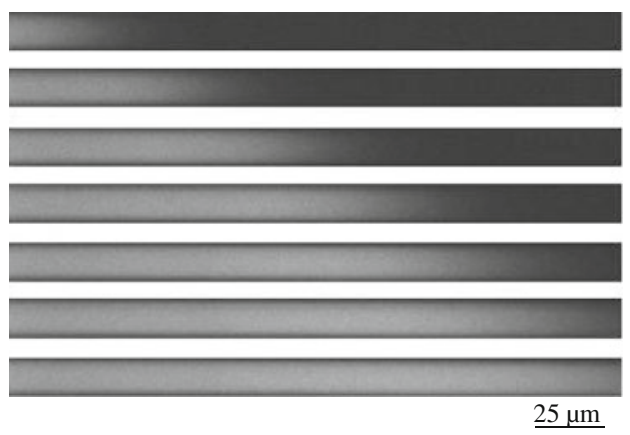

(b)

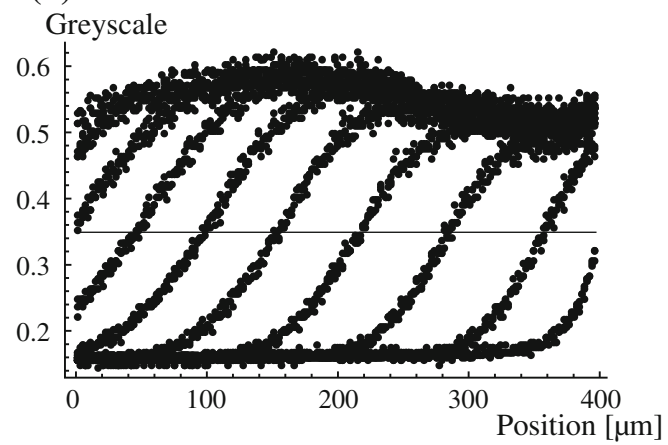

Fig. 16 a Fluorescent microscope image of the flow of fluorescein solution into the fluidic system replacing deionized water. Images taken with a time difference of $200 \mathrm{~ms}$ in a $400 \mu \mathrm{m}$ long microchannel. b Greyscale distribution in the center of the microchannel deduced out of a

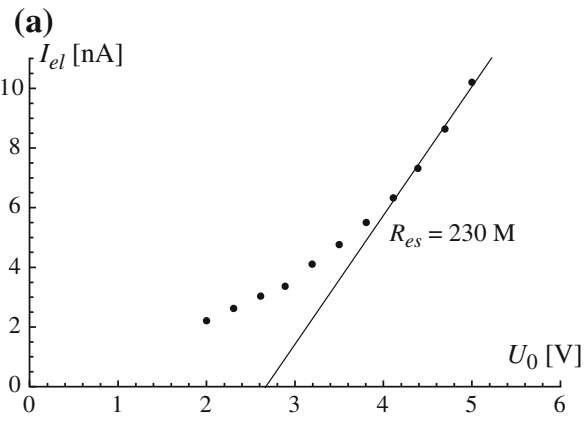

Fig. 17 a Voltage current curve of the eo pump. At low voltages the system is mainly determined by the current coupling of the electrode. The higher the applied voltage the higher the voltage drop over the fluidic system. The curve approaches the Ohmic behavior of the solution inside the fluidic system. b Current dependency of the flow into the eo pump $Q_{\text {in }}$ and the flow out of the eo pump $Q_{\text {out }}$. At zero eo

of the electrodes. The nonlinearity in this graph was caused by the current coupling of the electrode from the Pt into the solution. The consequence for the eo pump was, that for small currents, most of the potential drop occurred within the current coupling of the electrode and not over the eo pump. With higher currents, the electrical resistance of the eo pump became more dominant and, hence, most of the applied voltage dropped over the eo pump. This can also be seen, by the curve converging towards a linear, Ohmic behavior. To estimate the electrical resistance of the solution, the slope at a high potential, at $4.7 \mathrm{~V}$, was determined. The electrical resistance of the solution was determined to be $R_{e s}=230 \mathrm{M} \Omega$. With the geometry, the specific resistance of the solution was calculated to be $\rho_{e l}=100 \Omega \mathrm{m}$. This was contradicting to the initial specific resistance of the deionized water, but could be explained by minute liquid volume inside the fluidic system, where already small saline contamination had a strong effect on the specific resistance. In order to understand this decreased specific resistance of the solution, the kind and concentration of contaminating ions was estimated. The source of the

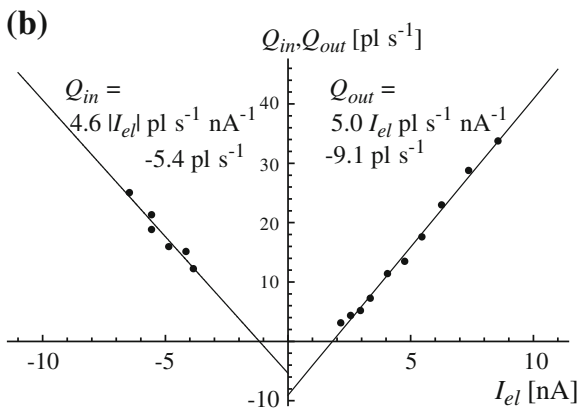

current the median of the two flows was induced by evaporation through the exhaust microchannels. The difference between the two flows was induced by a hydrostatic pressure between the two reservoirs connected to the in and outlet of the eo pump. The different slopes of the flows were introduced by misalignment of the electrodes within the lg-separator

contamination was speculated to be the residues of etching during fabrication and dissolved ions from the doped anodic bonding glass. Furthermore, the contamination is expected to be more of a saline character than an organic one, since the chips were cleaned in an oxygen plasma right before usage. Another observation supports this assumption, no local variations of the contact angle $\theta$ inside the microchannel were detected, which usually are a good indicator of organic contaminations. To assess the ionic concentration $c$ of the contaminants, Kohlrausch's law and Ostwald's law of dilution was used. A solution's specific resistance of $100 \Omega \mathrm{m}$ can be reached with an ionic concentration of $c=1 \mathrm{mM}$, based on an average limiting molar ionic conductivity of $100 \mathrm{~S} \mathrm{~cm}^{-2} \mathrm{~mol}^{-1}$ for a saline contamination (alkali metals, values for the average taken from Coury (1999)). In other terms, to reach this ionic concentration a surface contamination in the range of $5 \mu \mathrm{mol} \mathrm{m}{ }^{-2}$ was needed.

Knowing the ionic concentration $c$ of the solution allowed to calculate the eo mobility $\mu_{e o}$. The ionic concentration $c$ led to a Debye layer thickness of $\lambda_{D}=10 \mathrm{~nm}$ 
Table 2 Flows within the fluidic system

\begin{tabular}{|c|c|c|c|}
\hline & Measured & Modeled & \\
\hline Evaporation induced flow $v$ & $7.2 \mathrm{pl} \mathrm{s}^{-1}$ & $7.4 \mathrm{pl} \mathrm{s}^{-1}$ & \\
\hline Hydrostatic pressure $p_{h y}$ induced flow & $3.7 \mathrm{pl} \mathrm{s}^{-1}$ & & $p_{h y}=1.7 \mathrm{~Pa}$ \\
\hline Electrode misalignment $\varepsilon$ induced flow & $0.4 \mathrm{pl} \mathrm{s}^{-1} \mathrm{nA}^{-1}$ & $0.5 \mathrm{pl} \mathrm{s}^{-1} \mathrm{nA}^{-1}$ & Misalignment $2 \mu \mathrm{m}$ \\
\hline Flow into eo pump $Q_{\text {ineo }}$ & $4.6 \mathrm{pl} \mathrm{s}^{-1} \mathrm{nA}^{-1}$ & $4.6 \mathrm{pl} \mathrm{s}^{-1} \mathrm{nA}^{-1}$ & \\
\hline Flow out of eo pump $Q_{\text {outeo }}$ & $5.0 \mathrm{pl} \mathrm{s}^{-1} \mathrm{nA}^{-1}$ & $5.1 \mathrm{pl} \mathrm{s}^{-1} \mathrm{nA}^{-1}$ & \\
\hline
\end{tabular}

on a charged $\mathrm{SiO}_{2}$ surface. The surface charge $\sigma_{c h}$ at neutral $\mathrm{pH}$ values was determined by Zhou and Foley (2006) to $\sigma_{c h}=0.026 \mathrm{C} \mathrm{m}^{-2}$. Finally, this can be used to estimate the eo mobility to $\mu_{e o}=3 \times 10^{-7} \mathrm{~m}^{2} \mathrm{~V}^{-1} \mathrm{~s}^{-1}$. These assumptions consider only bulk conduction, according to Stein et al. (2004) an enhancement of the conductance due to electroosmosis plays an important role in nanochannels. In their findings, conduction only determined by the bulk can be assumed at low saline concentrations $c$ once: $w / \lambda_{D} \gg 1$ and $\left|\sigma_{c h}\right| \ll e c w$, where $e$ represents the electron charge. In second case, of the surface charge $\sigma_{c h}$, this is at its limit and, hence, the concentration $c$ might be slightly lower than estimated. Nevertheless, the values in this model are coarse estimations and treated as such, therefore, we do not expect a significantly different model. Moreover, the here derived values of the Debye layer thickness $\lambda_{D}$ and the eo mobility $\mu_{e o}$ were in good correspondence with those given by Wang et al. (2006).

The experimental result for the flow $Q_{\text {out }}$ out of the pump and the flow $Q_{\text {in }}$ into of the eo pump are shown in Fig. $17 \mathrm{~b}$ and summarized in Table 2. The evaporation induced flow $v$, was determined to be $v=7.2 \mathrm{pl} \mathrm{s}^{-1}$, according to Eq. 17. The modeled value for evaporation induced flow was in the range of $7.4 \mathrm{pl} \mathrm{s}^{-1}$. This value is based on the model developed in chapter 3 , with an evaporation area of two times $9 \times 20 \mu \mathrm{m}$ at a temperature of $20^{\circ} \mathrm{C}$ and a relative lab humidity of $33 \%$. The hydrostatic pressure $p_{\text {hy }}$ induced flow was determined to be $3.7 \mathrm{pl} \mathrm{s}^{-1}$, according to Eq. 18. The hydrostatic pressure $p_{h y}$ was related to a height difference of the meniscus in the pump inlet reservoir with respect to the meniscus in the pump outlet reservoir. From Eq. 18 a hydrostatic pressure difference of $p_{h y}=1.7 \mathrm{~Pa}$ was calculated. This corresponds to a height difference of the meniscus in the inlet reservoir of $0.17 \mathrm{~mm}$, lower than in the outlet reservoir. The misalignment of the electrodes was optically determined to be $2 \mu \mathrm{m}$ into the exhaust microchannel of the pump inlet. The induced eo flow difference between $Q_{\text {out }}$ and $Q_{i n}$ was determined to be $0.4 \mathrm{pl} \mathrm{s}^{-1} \mathrm{nA}^{-1}$, according to Eq. 19. Using the model and including the values for the specific resistance, the eo mobility $\mu_{e o}$ and a $2 \mu \mathrm{m}$ electrode misalignment led to an eo flow of $0.5 \mathrm{pl} \mathrm{s}^{-1} \mathrm{nA}^{-1}$. Finally, the general eo induced flow was determined to be $Q_{\text {ineo }}=4.6 \mathrm{pl} \mathrm{s}^{-1} \mathrm{nA}^{-1}$ and $Q_{\text {ou }}$ teo $=5.0 \mathrm{pl} \mathrm{s}^{-1} \mathrm{nA}^{-1}$, which is in good agreement with the modeled values of $Q_{\text {ineo }}=4.6 \mathrm{pl} \mathrm{s}^{-1} \mathrm{nA}^{-1}$ and $Q_{\text {outeo }}=5.1 \mathrm{pl} \mathrm{s}^{-1} \mathrm{nA}^{-1}$.

\section{Summary and conclusion}

To summarize, a successful analytically modeling and realization of an eo pump was shown. The novel approach of implementing a lg-separator, based on a tapered microchannel, allowed the current coupling from the $\mathrm{Pt}$ electrode into solution without mixing the emerging electrolyzed gases with the pumping liquid. Likewise, an easy fabrication technique based on a standard MEMS process and anodic bonding can be employed. All this resulted in an eo pump with a very small footprint of $100 \mu \mathrm{m} \times 15 \mu \mathrm{m}($ length $\times$ width $)$ and an actuation with a low voltage, $2 \mathrm{~V}-5 \mathrm{~V}$.

The lg-separator had a maximum backpressure of $7 \mathrm{kPa}$ and was able to reliably conduct away gas flows in the range of $2 \mathrm{pl} \mathrm{s}^{-1}$ generated at driving currents of up to $20 \mathrm{nA}$. The eo pump was modeled and measured. For deionized water, a flow out of the eo pump of $Q_{\text {out }}=5.0 \mathrm{pl} \mathrm{s}^{-1} \mathrm{nA}^{-1}$ was achieved. At an applied voltage of $5 \mathrm{~V}$, a current of $10 \mathrm{nA}$ was measured which amounts to a flow of $50 \mathrm{pl} \mathrm{s}^{-1}$. The developed model for the fluidic system also integrated effects like hydrostatic pressure and evaporation, as well as, fabrication imperfections like misalignment of the electrodes.

For a more detailed understanding of the electrode behavior a wider range of applied potentials is necessary. This would also allow a better prediction of the solutions specific conductivity and, hence, the eo mobility. In order to increase the backpressure of the eo pump an optimization can be achieved by connecting these pumps in series to obtain a multistage eo pump, as suggested by Takamura et al. (2003). Special attention needs to be taken in the design of the exhaust microchannels, since they will have to compensate for the increasing pressure between the 
stages. Moreover, a promising direction for further investigation would be to specifically use the Pt electrodes as integrated electrochemical sensors by optimizing the microchannel design accordingly and removing the emerging gas bubbles respecting the model of the lg-separator.

Acknowledgments The authors gratefully thank P.M. Sarro, C. de Boer, and L. Mele (DIMES-ECTM, Delft University of Technology) for their support in fabricating the microfluidic devices. The work presented, here, was partially funded by DCMM, Delft Centre for Mechatronics and Microsystems.

Open Access This article is distributed under the terms of the Creative Commons Attribution Noncommercial License which permits any noncommercial use, distribution, and reproduction in any medium, provided the original author(s) and source are credited.

\section{References}

Brask A, Kutter JP, Bruus H (2005) Long-term stable electroosmotic pump with ion exchange membranes. Lab Chip 5:730-738

Bruus H (2008) Theoretical microfluidics. Oxford University Press, Oxford

Concus P, Finn R (1969) On the behavior of a capillary surface in a wedge. Proc Natl Acad Sci 63:292-299

Coury L (1999) Conductance measurements part 1: theory. Curr Sep 18:91-96

Ghosal S (2004) Fluid mechanics of electroosmotic flow and its effect on band broadening in capillary electrophoresis. Electrophoresis 25:214-228

Guenat OT, Ghiglione D, Morf WE, de Rooij NF (2001) Partial electroosmotic pumping in complex capillary systems. Part 2: fabrication and application of a micro total analysis system ( $\mu$ tas) suited for continuous volumetric nanotitrations. Sens Actuators B 72:273-282

Guenther A, Jhunjhunwala M, Thalmann M, Schmidt MA, Jensen KF (2005) Micromixing of miscible liquids in segmented gas-liquid flow. Langmuir 21:1547-1555
Han A, Mondin G, Hegelbach NG, de Rooij NF, Staufer U (2006) Filling kinetics of liquids in nanochannels as narrow as $27 \mathrm{~nm}$ by capillary force. J Colloid Interface Sci 293:151-157

Hug TS, de Rooij NF, Staufer U (2005) Fabrication and electroosmotic flow measurements in micro- and nanofluidic channels. Microfluid Nanofluid 2:117-124

Jensen MJ, Goranovic G, Bruus H (2004) The clogging pressure of bubbles in hydrophilic microchannel contractions. J Micromech Microeng 14:876-883

Laser DJ, Santiago JG (2004) A review of micropumps. J Micromech Microeng 14:R35-R64

Lee TMH, Lee DHY, Liaw CYN, Lao AIK, Hsing IM (2000) Detailed characterization of anodic bonding process between glass and thin-film coated silicon substrates. Sens Actuators A 86:103-107

Madou MJ (2002) Fundamentals of microfabrication, 2nd edn. CRC Press, Boca Raton

Morf WE, Guenat OT, de Rooij NF (2001) Partial electroosmotic pumping in complex capillary systems. Part 1: principles and general theoretical approach. Sens Actuators B 72:266-272

Paust N, Litterst C, Metz T, Eck M, Ziegler C, Zengerle R, Koltay P (2009) Capillary-driven pumping for passive degassing and fuel supply in direct methanol fuel cells. Microfluid Nanofluid 7:531-543

Plaza JA, Esteve J, Lora-Tamayo E (1998) Effect of silicon oxide, silicon nitride and polysilicon layers on the electrostatic pressure during anodic bonding. Sens Actuators A 67:181-184

Seibel K, Schoeler L, Schaefer H, Boehm M (2008) A programmable planar electroosmotic micropump for lab-on-a-chip applications. J Micromech Microeng 18. doi:0.1088/0960-1317/18/025008

Stein D, Kruithof M, Dekker C (2004) Surface-charge-governed ion transport in nanofluidic channels. Phys Rev Lett 93. doi: 10.1103/PhysRevLetter.93.035901

Takamura Y, Onoda H, Inokuchi H, Adachi S, Oki A, Horiike Y (2003) Low-voltage electroosmosis pump for stand-alone microfluidics devices. Electrophoresis 24:185-192

Wang P, Chen Z, Chang HC (2006) A new electro-osmotic pump based on silicamonoliths. Sens Actuators B 113:500-509

White FM (1999) Fluid mechanics, 4th edn. McGraw-Hill, New York

Zhou MX, Foley JP (2006) Quantitative theory of electroosmotic flow in fused-silica capillaries using an extended site-dissociationsite-binding model. Anal Chem 78:1849-1858 